\title{
1-506-3
}

\section{Nursing pharmacology and clinical practice}

\section{Takeshi Unoki}

School of Nursing, Sapporo City University

I have experienced for clinical practice in the intensive care unit for more than 10 years and am working as a professor in nursing school. In this symposium, I will talk about pharmacology education for nursing student and clinical nurses based on my experience.

The point of our opinion is pharmacology in nursing curriculum at this time is just pharmacology, not but pharmacology for nursing or nurses. Off course, pharmacology is pharmacology, however, its education should be changed depending on medical profession and it should contribute to knowledge in terms practice. It is time for transition from pharmacology education for pharmacology to pharmacology education for specifically nursing students and clinical nurses. 